\title{
Current Applications of Computational Chemistry in JACS-Molecules, Mechanisms, and Materials
}

$\mathrm{T}$ his virtual issue of JACS Select is devoted to some of the computational chemistry that has recently been published in the Journal of the American Chemical Society. The 25 Articles and Communications that appear in this issue of JACS Select were chosen on the basis of the enthusiasm of the referees for these manuscripts when they were reviewed, as well as on the number of times each of these articles has been accessed online since its publication.

Diversity of subject matter was not a criterion used in selecting these 25 articles from the ca. 50 well-reviewed, frequently accessed articles that were initially considered. However, inspection of the list of the subjects covered-from amyloid fibril polymorphism to nucleation of amorphous calcium carbonate; from the structure of solid $\mathrm{Li}\left(\mathrm{NH}_{3}\right)_{4}$ to the role that solvation plays in the Thorpe-Ingold effect; and to the nonstatistical, gasphase dynamics of $[1,5]$ hydrogen shifts in chemically activated cyclopentadiene-reveals the diverse nature of some of the best papers in computational chemistry that have been published recently in JACS.

It may come as a surprise to some JACS readers to learn that the computational chemistry articles that appear in JACS do not even begin to span the types of research that computational chemists do. Many computational chemists publish articles on improving existing computational methodology and/or creating new methods. A new or improved method must be tested to see how it compares in speed and efficiency with existing methodology and, most importantly, whether the new methods give results that are in better agreement with experiment than similar methods that are already in use. Many publications in computational chemistry are devoted to benchmarking computational methodology.

However, much to the dismay of some computational chemists, manuscripts that report method development and/or benchmarking are rarely accepted for publication in JACS. This is not a secret JACS Editorial policy; it is stated explicitly in the JACS Notice to Authors of Papers. ${ }^{1}$ This document reads, "Articles on methodology should include one or more applications of widespread interest and, unless significant new advances are announced, will be declined with the recommendation for submission to specialized journals. Papers that draw conclusions from the treatment of existing data must also include significant new data and make new experimental or theoretical predictions of broad interest."

Why are articles on methods development and benchmarking not published by JACS? There are several reasons for this JACS policy. Perhaps the most important is that "broad interest" is an important criterion that all manuscripts must satisfy in order to be published in JACS. Experimentalists constitute the overwhelming majority of JACS readers, and although experimentalists may be quite interested in reading computational manuscripts that provide new insights into molecules, mechanisms, or materials, most experimentalists are much less interested in reading about new advances in computational methodology, at least until the power and usefulness of the methodology has been demonstrated. No matter how promising a new computational method may appear initially, whether the method will actually prove useful is usually not apparent until the method has been tested on a wide variety of molecules and reactions, or until it has been used to perform a calculation of "widespread interest" that has not previously been practical.

Some theoretical chemists disagree with JACS's general policy against publication of new computational methodologies in the Journal. These theoreticians sometimes argue that, if a new method turns out to be very important, then not publishing the method in JACS will delay the dissemination of the method to potential users. This assertion may, in fact, be true, but, if a new method really is useful, it will be only a short while before computational chemists discover it and begin submitting manuscripts to JACS, reporting the use of the new method to solve chemical problems.

Publications on computational methodology or benchmarking will not be found in this issue of JACS Select. Instead, this issue contains 25 first-rate Articles and Communications on the application of calculations to chemical research, ranging from molecules to mechanisms to materials.

It may prove worthwhile for readers of this issue of JACS Select, especially readers who are contemplating submitting a computational manuscript of their own to JACS, to examine these publications in order to see how each of them fulfills the three requirements for a computational manuscript to be published in JACS. Again quoting from the 2011 JACS Notice to Authors of Papers, "It is required [for computational manuscripts] that (a) the level of theory and methodology employed must be adequate for the problem at hand, (b) theoretical findings must be strongly correlated with experimental observables and relevant experimental data (to the extent available), and (c) the manuscript must provide significant chemical insights and have substantial predictive value."

The reason for the first requirement is obvious. The second emphasizes the importance of tying computational manuscripts that are published in JACS to experiments that have already been done. The third requirement is that, either implicitly or explicitly, a manuscript should make some predictions about the outcomes of experiments that have not yet been performed.

A manuscript may convincingly explain all of the existing experimental data in an area of chemistry, but an explanation that cannot be tested by additional experiments is actually of little use. The best computational manuscripts motivate new experiments. The author essentially says to the reader, "If you do the following experiment, I predict this is exactly what you will find." The integration of calculations with experimental results and predictions is evident to different degrees in all of the Articles and Communications in this issue of JACS Select.

Published: September 21, 2011 
Aikens and co-workers use calculations to predict the structure of a thiolate-protected $\mathrm{Au}_{38}$ nanocluster. The properties computed for the predicted structure are found to be in very good agreement with those that have been measured. ${ }^{2}$ Buchwald, Houk, and co-workers investigate computationally the reason for the dependence on catalyst structure of the regioselectivity of $\mathrm{N}$ versus $\mathrm{O}$ in Ullmann-type reactions and provide a convincing rationalization. ${ }^{3}$

Caflisch and co-workers report the results of molecular dynamics (MD) simulations which find that amyloid polymorphism is under kinetic rather than thermodynamic control. ${ }^{4}$ On the basis of the results of another type of MD simulation, semiclassical trajectory calculations, Carpenter, Glowacki, and Goldman make a bold prediction about the ring-opening of bicyclo[2.1.0]pentene. Their calculations indicate that whether the initially exo or endo hydrogen subsequently migrates should show an oscillatory dependence on time, and the authors propose an experiment by which this intriguing prediction might be tested..$^{5}$ Cecchini, Samorì, and Palma also report on the results of MD calculations, in this case to simulate the self-assembly of two-dimensional structures, formed on graphite, from hydrogen-bond donors and hydrogen-bond acceptors. ${ }^{6}$

Coote, Easton, and Ho describe the use of electronic structure calculations to predict that substituents on nitrogen have a strong effect on the acidities of the protons attached to the carbons that are alpha to amide carboxyl groups. The authors report the results of preliminary experiments that appear to confirm this prediction. ${ }^{7}$ Deumal, Novoa, and co-workers compute the radical-radical interactions in two crystalline phases of the 1,3,5-trithia-2,4, 6-triazapentalenyl radical, in order to understand why the hightemperature phase is paramagnetic, whereas the low-temperature phase is diamagnetic.

Driess, Apeloig, Karni, and co-workers describe a combined computational and experimental study of the nature of the $\mathrm{Si}=\mathrm{O}$ and $\mathrm{Si}=\mathrm{S}$ double bonds in silanoic and thiosilanoic esters. ${ }^{9}$ Eisenstein, Crabtree, Brudvig, and co-workers report another combined computational/experimental study, this one focused on the factors that control the switch between hydroxylation and desaturation of the radical intermediates that are formed by $\mathrm{Mn}(\mathrm{V})=\mathrm{O}$ oxidation reactions. ${ }^{10}$

Raiteri and Gale use MD to simulate the formation of amorphous calcium carbonate nanoparticles, which serve as the precursors to crystalline polymorphs of $\mathrm{CaCO}_{3}$ and lead to biomineralization. ${ }^{11}$ Hammes-Schiffer and Chakravorty use MD for a very different purpose-to understand the proton-transfer step in the mechanism of action of the enzyme ketosteroid isomerase. ${ }^{12}$

Zureck and co-workers perform density functional calculations on the very low-melting, "expanded metal", $\mathrm{Li}\left(\mathrm{NH}_{3}\right)_{4}$, in the solid state. ${ }^{13}$ Houk and Lan also use density functional calculations, but with a very different goal, to investigate the factors that make the reaction of a carbonyl ylide with an electron-deficient alkene in solution a rare example of a nonconcerted 1,3-dipolar cycloaddition reaction. ${ }^{14}$

Although Eric Jacobsen is much better known for his experimental work on enantioselective catalysis than for his research in computational chemistry, Jacobsen and Uyeda have taken advantage of density functional theory (DFT) calculations in order to design a new guanidinium catalyst for an enantioselective Claisen rearrangement. The authors report successful experimental tests of their new catalyst. ${ }^{15}$

In a purely computational publication, Jorgensen and Kostal report the results of Monte Carlo statistical mechanics calculations on the rate of oxirane formation from 2-chloroethanol in aqueous solution. Their simulations show that the Thorpe-Ingold rate acceleration observed on adding a pair of methyl groups to C-1 of the reactant is largely due not to relief of strain in the transition structure, but rather to steric inhibition of solvation of the nucleophilic oxygen. ${ }^{16}$ Moss, Krogh-Jespersen, and co-workers report a combined computational and experimental study of the complexes formed between phenylchlorocarbene and 1,3, 5-trimethoxybenzene. ${ }^{17}$

Morokuma and co-workers use DFT calculations and Morokuma's ONIOM method in order to investigate the regioand stereochemistry of the rhodium-catalyzed polymerization of phenylacetylene. ${ }^{18}$ In another study of how solid-state structure affects the magnetic properties of radicals, Oakley and co-workers describe the results of DFT calculations and experiments on the two crystalline forms of a bisthiazoyl radical. ${ }^{19}$ In yet another investigation of an organometallic reaction mechanism, Poli and Dub investigate the mechanism for the Pt-catalyzed hydroamination of ethylene by aniline. ${ }^{20}$

Solomon and co-workers report the results of a computational investigation of electron transport through $\pi$-stacked molecules. Interestingly, they find that they can relate their results to the topologies of the interactions that produce singlet and triplet ground states in the analogous diradicals. ${ }^{21}$

Singleton and Gonzalez-James combine DFT calculations with kinetic isotope effect measurements to establish that $\mathrm{CO}_{2}$ loss is rate determining in the decarboxylation of mandelylthiamin. Their calculations also solve the mystery of how pyridinium ions catalyze the decarboxylation reaction if $\mathrm{CO}_{2}$ loss is rate determining. ${ }^{22}$

Stoychev and co-workers perform calculations on intermolecular Coulombic decay (ICD) in small hydrogen-bonded molecules, in order to model the effect of ICD on hydrogen-bonded biomolecules. ${ }^{23}$ Szleifer and co-workers report the results of their simulations of fluid flow through a nanoscale channel, lined with a polyelectrolyte brush. They find that the results of their simulations are in excellent agreement with the experimental results on transport through polymer-lined nanochannels. ${ }^{24}$

Truhlar and co-workers use density functional calculations, including a model for solvent effects, to investigate elimination reactions that lie close to the border between concerted E2 and stepwise E1cB mechanisms. ${ }^{25}$ Finally, Yang, McCafferty, and coworkers combine quantum mechanics with molecular mechanics (QM/MM) to investigate the autocatalytic, isopeptide bond formation involved in the pili of Gram-positive bacteria. ${ }^{26}$

What generalizations can be made about such a diverse group of publications in computational chemistry? One obvious generalization is that calculations are currently being used to investigate a very wide range of phenomena in chemistry. A second generalization is that calculations are no longer limited, as they once were, just to exploring the structures and reactions of comparatively small molecules in the gas phase. The use of DFT and $\mathrm{QM} / \mathrm{MM}$ methods enables calculations to be performed on very large molecules, and most of the calculations on reaction mechanisms in this JACS Select collection simulate the mechanisms in solution, where the reactions actually were carried out.

From a methodological perspective, the Articles and Communications in this issue of JACS Select indicate that calculations based on DFT have largely supplanted $a b$ intio calculations in the majority of computational work that is published in the Journal. Judging from the number of papers in this virtual issue that report the results of $\mathrm{MD}$ simulations, the popularity of this type of simulation for studying structure and reactivity is currently on the rise. 
To someone who believes strongly in the synergism between calculations and experiments, what is most striking about the 25 contributions in this issue of JACS Select is the close coupling between these two types of research activity. Most of the computational research described in these publications was initiated in order to understand the results of previously published experiments and/or to help analyze the results of experiments that are reported in the same article.

Some three decades ago, Jerry Berson, one of the giants of experimental physical-organic chemistry of the past century, remarked, "Experimentalists ignore the results of calculations at their own peril." Jerry was one of the first experimentalists to appreciate the role that calculations can play in experimental research, and, after reading the 25 computational publications in this issue of JACS Select, today Jerry might have said, "Experimentalists ignore the power of current computational chemistry to the detriment of their own research."

Perhaps the 25 computational publications in this issue will persuade some experimentalists to collaborate with computational chemists on one or more research projects. In order to take maximal advantage of calculations as a powerful tool for chemical research, other experimentalists may be motivated to learn enough about computational chemistry to be able to do calculations themselves. A glossary for nonexperts of terms and concepts commonly used in computational chemistry accompanies this JACS Select collection on the Web.

\section{Weston Thatcher Borden \\ Associate Editor}

\section{REFERENCES}

(1) This useful guide may be read on or downloaded from the Web by visiting http://pubs.acs.org/page/jacsat/submission/authors.html and clicking on "Author Guidelines [PDF]".

(2) Lopez-Acevedo, O.; Tsunoyama, H.; Tsukuda, T.; Häkkinen, H.; Aikens, C. M. J. Am. Chem. Soc. 2010, 132, 8210.

(3) Jones, G. O.; Liu, P.; Houk, K. N.; Buchwald, S. L. J. Am. Chem. Soc. 2010, 132, 6205.

(4) Pellarin, R.; Schuetz, P.; Guarnera, E.; Caflisch, A. J. Am. Chem. Soc. 2010, 132, 14960.

(5) Goldman, L. M.; Glowacki, D. R.; Carpenter, B. K. J. Am. Chem. Soc. 2011, 133, 5312.

(6) Palma, C.-A.; Samorì, P.; Cecchini, M. J. Am. Chem. Soc. 2010, 132,17880 .

(7) Ho, J.; Easton, C. J.; Coote, M. L. J. Am. Chem. Soc. 2010, 132, 5515.

(8) Clarke, C. S.; Jornet-Somoza, J.; Mota, F.; Novoa, J. J.; Deumal, M. J. Am. Chem. Soc. 2010, 132, 17817.

(9) Epping, J. D.; Yao, S.; Karni, M.; Apeloig, Y.; Driess, M. J. Am. Chem. Soc. 2010, 132, 5443.

(10) Hull, J. F.; Balcells, D.; Sauer, E. L. O.; Raynaud, C.; Brudvig, G. W.; Crabtree, R. H.; Eisenstein, O. J. Am. Chem. Soc. 2010, 132, 7605.

(11) Raiteri, P.; Gale, J. D. J. Am. Chem. Soc. 2010, 132, 17623.

(12) Chakravorty, D. K.; Hammes-Schiffer, S. J. Am. Chem. Soc. 2010, 132, 7549.

(13) Zurek, E.; Wen, X.-D.; Hoffmann, R. J. Am. Chem. Soc. 2011, 133, 3535 .

(14) Lan, Y.; Houk, K. N. J. Am. Chem. Soc. 2010, 132, 17921.

(15) Uyeda, C.; Jacobsen, E. N. J. Am. Chem. Soc. 2011, 133, 5062.

(16) Kostal, K.; Jorgensen, W. L. J. Am. Chem. Soc. 2010, 132, 8766.

(17) Moss, R. A.; Wang, L.; Odorisio, C. M.; Krogh-Jespersen, K. J. Am. Chem. Soc. 2010, 132, 10677.

(18) Ke, Z.; Abe, S.; Ueno, T.; Morokuma, K. J. Am. Chem. Soc. 2011, 133,7926
(19) Lekin, K.; Winter, S. M.; Downie, L. E.; Bao, X.; Tse, J. S.; Desgreniers, S.; Secco, R. A.; Dube, P. A.; Oakley, R. T. J. Am. Chem. Soc. 2010, 132, 16212.

(20) Dub, P. A.; Poli, R. J. Am. Chem. Soc. 2010, 132, 13799.

(21) Solomon, G. C.; Herrmann, C.; Vura-Weis, J.; Wasielewski, M. R.; Ratner, M. A. J. Am. Chem. Soc. 2010, 132, 7887.

(22) Gonzalez-James, O. M.; Singleton, D. A. J. Am. Chem. Soc. 2010, 132, 6896.

(23) Stoychev, S. D.; Kuleff, A. I.; Cederbaum, L. S. J. Am. Chem. Soc. 2011, 133, 6817.

(24) Tagliazucchi, M.; Azzaroni, O.; Szleifer, I. J. Am. Chem. Soc. 2010, 132, 12404.

(25) Kim, Y.; Mohrig, J. R.; Truhlar, D. G. J. Am. Chem. Soc. 2010, $132,11071$.

(26) Hu, X.; Hu, H.; Melvin, J. A.; Clancy, K. W.; McCafferty, D. G.; Yang, W. J. Am. Chem. Soc. 2011, 133, 478. 